\title{
Nonperturbative effects on radiative energy loss of heavy quarks
}

\author{
Shuai Y.F. Liu ${ }^{a, b}$ and Ralf $\operatorname{Rapp}^{a}$ \\ ${ }^{a}$ Cyclotron Institute and Department of Physics and Astronomy, Texas A\&M University, \\ MS 3366, College Station, TX 77843-3366, U.S.A. \\ ${ }^{b}$ Quark Matter Research Center, Institute of Modern Physics, Chinese Academy of Sciences, \\ 509 Nanchang Rd., Lanzhou, Gansu, 730000, China \\ E-mail: 1shphy@gmail.com, rapp@comp.tamu.edu
}

ABSTRACT: The radiative energy loss of fast partons traveling through the quark-gluon plasma (QGP) is commonly studied within perturbative QCD (pQCD). Nonperturbative (NP) effects, which are expected to become important near the critical temperature, have been much less investigated. Here, we utilize a recently developed $T$-matrix approach to incorporate NP effects for gluon emission off heavy quarks propagating through the QGP. We set up four cases that contain, starting from a Born diagram calculation with colorCoulomb interaction, an increasing level of NP components, by subsequently including (remnants of) confining interactions, resummation in the heavy-light scattering amplitude, and off-shell spectral functions for both heavy and light partons. For each case we compute the power spectra of the emitted gluons, heavy-quark transport coefficients (drag and transverse-momentum broadening, $\hat{q}$ ), and the path-length dependent energy loss within a "QGP brick" at fixed temperature. Investigating the differences in these quantities between the four cases illustrates how NP mechanisms affect gluon radiation processes. While the baseline perturbative processes experience a strong suppression of soft radiation due to thermal masses of the emitted gluons, confining interactions, ladder resummations and broad spectral functions (re-)generate a large enhancement toward low momenta and low temperatures. For example, for a $10 \mathrm{GeV}$ charm quark at $200 \mathrm{MeV}$ temperature, they enhance the transport coefficients by up to a factor of 10, while the results smoothly converge to perturbative results at sufficiently hard scales.

KeYwords: Heavy Ion Phenomenology, Jets

ARXIV EPRINT: 2003.12536 


\section{Contents}

1 Introduction 1

2 Gluon radiation in the $\boldsymbol{T}$-matrix formalism 3

2.1 Many-body formalism of heavy-quark radiative energy loss 3

$\begin{array}{lll}2.2 & \text { Relation to pQCD diagrams } & 6\end{array}$

$\begin{array}{lll}2.3 & \text { Four cases of NP effects } & 7\end{array}$

3 Numerical analysis $\quad 9$

3.1 Spectral properties of radiated partons 9

$\begin{array}{lll}3.2 & \text { Power spectra } & 10\end{array}$

$\begin{array}{lll}3.3 & \text { Drag coefficient and energy loss } & 15\end{array}$

$\begin{array}{ll}3.4 & \text { Comparison to a pQCD calculation } \\ \end{array}$

$\begin{array}{llr}4 \text { Conclusion } & 19\end{array}$

\section{Introduction}

In ultra-relativistic heavy-ion collisions (URHICs) heavy quarks are mostly produced in primordial hard collisions of the incoming nucleons, on a short timescale, $\tau_{\text {prod }} \sim 1 / 2 m_{Q}$, governed by the heavy-quark (HQ) mass, $m_{Q}$; therefore, they probe the entire evolution of the fireball formed in these reactions (a sizeable secondary production of HQ pairs may occur at the Future Circular Collider (FCC) [1]) . The large mass of heavy quarks also enables theoretical simplifications in the description of their transport through the medium [2], allowing for rather direct connections between their microscopic interactions and pertinent observables in experiment. Heavy-flavor (HF) hadrons are therefore excellent probes of the properties of the QGP and its hadronization in URHICs [2-6].

One generally distinguishes two processes that control the dynamics of heavy quarks in the QGP - collisional and radiative ones. The former are mainly responsible for thermalization of heavy quarks at low momenta, $p_{Q} \simeq \sqrt{3 M_{Q} T}$, while the latter become increasingly relevant at higher $\mathrm{HQ}$ momenta [7-9]. Radiative processes, i.e., medium-induced gluon emission, in the relativistic limit have been widely studied over the past two decades. Various formalisms, such as AMY [10], ASW [11], BDMPS-Z [12, 13], DGLV [14, 15], higher-twist [16, 17], and SCET [18, 19], have been developed for the phenomenology of light-flavor jet quenching in URHICs. Among them, some have been extended to massive quarks [11, 20-24] and implemented into numerical simulations for their transport in URHICs [8, 25-30], see also refs. [31] and [32, 33] for recent comparisons between different energy loss formalisms for light-flavor jets and heavy quarks, respectively. Gluon emission within these approaches is generally described using perturbative-QCD (pQCD) 
methods. Within different expansion schemes, these approaches concentrate on the regions of the phase space where nonperturbative (NP) effects are expected to be small or can be absorbed into a transport parameter that encodes the medium properties, most notably the jet transport coefficient, $\hat{q}$. So far, few studies of NP effects in radiative processes have been carried out at a microscopic level (see, e.g., ref. [27]), especially in the relatively low-momentum and -temperature and temperature regimes where standard expansion and factorization schemes are no longer reliable. The objective of the present work is to utilize a NP T-matrix approach to provide insights into mechanisms of radiative energy loss of heavy quarks which are not easily assessed in $\mathrm{pQCD}$ approaches.

The phenomenology of HF observables in URHICs, especially the measured elliptic flow, requires heavy quarks to have an interaction strength with the medium that goes well beyond pQCD [4, 32, 34], especially at low momentum and temperature. As rigorous QCD calculations are challenging in this regime, one has to resort to model approaches. An essential step in improving their reliability is to root the model parameters in fits to lattice-QCD (1QCD) data as much as possible. In our previous work we have developed a quantum many-body approach with a QCD-inspired model Hamiltonian [35-37] that turns out to be an effective tool in describing a wide variety of QGP properties with a relatively small number of parameters, including its equation of state (EoS), HQ free energies, and correlation functions, while predicting spectral properties and transport coefficients (e.g., shear viscosity and HQ diffusion coefficient). Key features of this approach are the inclusion of remnants of the confining force in the QGP, ladder resummed amplitudes leading to the dynamical generation of bound states as the pseudo-critical temperature, $T_{\mathrm{pc}}$, is approached from above, and a full off-shell treatment of propagators and scattering amplitudes which is, in fact, mandated by the large widths that develop for low-momentum partons. In this work, we will deploy this approach to calculate the radiative energy loss of heavy quarks. The off-shell transport theory underlying this framework is based on the Kadanoff-Baym equation following the same logic as used in our previous study of the HQ collisional energy loss [37]. To illustrate how NP physics affects gluon radiation, we set up a systematic comparison between four different scenarios. Case (1) is the perturbative baseline in our approach that only includes screened Coulomb interactions at the Born level. We add confining interactions in case (2) and the resummation of $t$-channel diagrams in case (3). In case (4), we further include the off-shell effects for parton and HQ propagators, i.e., their broad spectral functions, representing the full results in our current framework. We analyze the differences between these four setups for several quantities, such as emission power spectra, transport coefficients, and fractional energy loss within a QGP brick medium.

The paper is organized as follows. In section 2 we lay out our formalism for calculating the HQ radiative energy loss within the $T$-matrix formalism (section 2.1), discuss its relation to pQCD diagrams (section 2.2), and define four different model setups with an increasing content of NP components (section 2.3). In section 3, we compare the numerical results for typical energy loss quantities from these four cases. A summary and outlook is given in section 4 . 


\section{Gluon radiation in the $\boldsymbol{T}$-matrix formalism}

In this section, we first develop the formalism for computing the radiative energy loss in the context of the in-medium $T$-matrix (section 2.1), discuss the relation of the NP calculation to the diagrams in pQCD including the issue of gauge invariance (section 2.2), and then introduce four different model cases of increasing levels of NP effects to illustrate how these influence radiative processes of charm quarks (section 2.3).

\subsection{Many-body formalism of heavy-quark radiative energy loss}

The derivation of the formalism for evaluating radiative processes is similar to that in our previous work [37]. We first recall the calculation for collisional processes and then derive the equations for radiative processes.

We start with a Kadanoff-Baym equation for the heavy quark $(Q)$ in terms of its Green's function, $G_{Q}$ and selfenergy, $\Sigma_{Q}$,

$$
\left.\frac{\partial}{\partial t}\left[\int d \omega G_{Q}^{<}(\omega, \mathbf{p}, t)\right]=\int d \omega\left[i \Sigma_{Q}^{<}(\omega, \mathbf{p}, t) G_{Q}^{>}(\omega, \mathbf{p}, t)-i \Sigma_{Q}^{>}(\omega, \mathbf{p}, t)\right) G_{Q}^{<}(\omega, \mathbf{p}, t)\right],
$$

where the $>,<$ superscripts denote the fixed-ordered quantities in real-time manybody field theory (sometimes referred to as Wightman functions). They are related to $G^{>/<}\left(t_{1}, t_{2}, x_{1}, x_{2}\right)$ in coordinate space through a Wigner transform over relative and total space-time, $\delta t=t_{1}-t_{2}, \delta \mathbf{x}=\mathbf{x}_{1}-\mathbf{x}_{2}$ and $t=\left(t_{1}+t_{2}\right) / 2, \mathbf{x}=\left(\mathbf{x}_{1}+\mathbf{x}_{2}\right) / 2$, respectively, where the explicit $\mathbf{x}$ dependence drops out due to our assumption of translational invariance. By putting the incoming heavy quark on-shell [37], eq. (2.1) can be reduced to a semi-classical Boltzmann equation for its phase space distribution (PSD) function, $f_{Q}$

$$
\frac{\partial}{\partial t} f_{Q}(\mathbf{p}, t)=\int \frac{d^{3} \mathbf{k}}{(2 \pi)^{3}}\left[w(\mathbf{p}+\mathbf{k}, \mathbf{k}) f_{Q}(\mathbf{p}+\mathbf{k}, t)-w(\mathbf{p}, \mathbf{k}) f_{Q}(\mathbf{p}, t)\right],
$$

where the transition rate $w(\mathbf{p}, \mathbf{k})$ encodes the quantum many-body information of the system, which can be used to obtain transport coefficients [3]. The first and second terms on the right hand side of eq. (2.2) correspond to the first and second terms of eq. (2.1) respectively, as discussed in ref. [37].

For the collisional $2 \rightarrow 2$ scattering processes, $\Sigma_{Q}^{>}$in eq. (2.1) can be schematically expressed via a HQ scattering amplitude off thermal partons, $M_{Q i \rightarrow Q i}$, as

$$
\Sigma_{Q}^{>}(p)=\int d^{4} \tilde{p}^{\prime} d^{4} \tilde{q} d^{4} \tilde{q}^{\prime}(2 \pi)^{4} \delta^{(4)}\left|M_{Q i \leftrightarrow Q i}\right|^{2} G_{Q}^{>}\left(p^{\prime}\right) G_{i}^{<}(q) G_{i}^{>}\left(q^{\prime}\right),
$$

where $p\left(p^{\prime}\right)$ and $q\left(q^{\prime}\right)$ are the 4-momenta of the incoming (outgoing) heavy quark and thermal parton, $i$, respectively. We use the notation $d^{4} \tilde{p}=d^{4} p /(2 \pi)^{3} 2 \epsilon(p)$ for the 4momentum phase space elements (with on-shell energy $\epsilon_{Q}(p)$ ) and $\delta^{(4)}$ for the 4-momentum conserving Dirac delta function. The Green's functions can be represented by the spectral functions $(\rho)$ with Bose/Fermi factors, $n_{i, Q}$, as

$$
\begin{aligned}
& G_{Q}^{>}(\omega, p)=-i(2 \pi) \rho_{Q}(\omega, p)\left(1-n_{Q}(\omega)\right), \\
& G_{i}^{<}(\omega, p)=\mp i(2 \pi) \rho_{i}(\omega, p) n_{i}(\omega), \\
& G_{i}^{>}(\omega, p)=-i(2 \pi) \rho_{i}(\omega, p)\left(1 \pm n_{i}(\omega)\right),
\end{aligned}
$$


The transition rate, $w(\mathbf{p}, \mathbf{k})$, derived from $\Sigma_{Q}^{>}$can then be expressed as

$$
w(\mathbf{p}, \mathbf{k})=\int d^{4} \tilde{q} d^{4} \tilde{q}^{\prime} d \omega^{\prime}(2 \pi)^{4} \delta^{(4)}\left|M_{Q i \leftrightarrow Q i}\right|^{2} \rho_{Q}\left[1-n_{Q}\right] \rho_{i} n_{i} \rho_{i}\left[1 \mp n_{i}\right],
$$

where $\omega^{\prime}$ is the energy of the outgoing heavy quark. The off-shell spectral functions of both thermal partons and heavy quarks $(x=Q, i)$, which encode the non-trivial quantum many-body physics of the system, can be expressed as

$$
\begin{aligned}
\rho_{x}(k)= & -\operatorname{Im}\left(\frac{1}{\omega-\varepsilon_{x}(p)-\Sigma_{x}(\omega, p)}\right) \\
\operatorname{Im} \Sigma_{x}(p)= & -\sum_{j} \int d^{4} \tilde{p}^{\prime} d^{4} \tilde{q} d^{4} \tilde{q}^{\prime}(2 \pi)^{4} \delta^{4}\left|M_{x j \leftrightarrow x j}\right|^{2} \rho_{j}(q) \rho_{j}\left(q^{\prime}\right) \rho_{x}\left(p^{\prime}\right) \\
& \times\left[n_{j}\left(1-n_{j}\right)\left(1-n_{x}\right)+\left(1-n_{j}\right) n_{x} n_{j}\right] \\
\equiv & \sum_{j} \int d^{4} \tilde{q} \operatorname{Im} M_{x j \rightarrow x j} \rho_{j}(q)\left(n_{j} \pm n_{j x}\right),
\end{aligned}
$$

where the $M_{x j \rightarrow x j}$ are "elastic" heavy-light or light-light scattering amplitudes. The summation is over all internal degrees of freedom with spin $(s)$, color $(c)$ and flavor $(f)$ degeneracy factors and averaging procedure. The last line of eq. (2.7) follows from the optical theorem, which we utilize to calculate the elastic selfenergy as detailed in ref. [36]. The real part of the selfenergy is obtained from a dispersion relation.

The key step to extend the above formalism from collisional to radiative processes is to replace the collisional amplitude $M_{2 \rightarrow 2}$ in eq. (2.3) with a radiative amplitude $M_{1 \rightarrow 2}$ and remove one incoming Green's function, $G_{i}^{<}$. This leads to

$$
\begin{aligned}
\Sigma_{Q}^{>}(p) & =\int d^{4} \tilde{p}^{\prime} d^{4} \tilde{k}(2 \pi)^{4} \delta^{4}\left|M_{Q \rightarrow Q g}\right|^{2} G_{Q}^{>} G_{g}^{>}, \\
w(\mathbf{p}, \mathbf{k}) & =\int d^{4} \tilde{p}^{\prime} d \nu(2 \pi)^{4} \delta^{(4)}\left|M_{Q \rightarrow Q g}\right|^{2} \rho_{Q}\left[1-n_{Q}\right] \rho_{g}\left[1+n_{g}\right],
\end{aligned}
$$

where $k=(\nu, \mathbf{k})$ is he outgoing gluon 4-momentum. The amplitude for the $Q \rightarrow Q g$ process is calculated using the pQCD diagrams for gluon emission,

$$
\begin{aligned}
\left|M_{Q \rightarrow Q g}\right|^{2} & \approx \sum_{c, s} g^{2}\left|\bar{u}\left(p^{\prime}\right) \gamma_{\mu} u(p) \epsilon^{\mu}(k)\right|^{2}= \\
& =4 d_{Q} C_{F} g^{2}\left\{\left(\varepsilon_{Q}(p) \varepsilon_{Q}\left(p^{\prime}\right)-m_{Q}^{2}\right)-\frac{(\mathbf{p} \cdot \mathbf{k})\left(\mathbf{p}^{\prime} \cdot \mathbf{k}\right)}{\mathbf{k}^{2}}\right\} .
\end{aligned}
$$

We choose the standard Dirac spinor $(u)$ and the polarization tensor $\left(\epsilon^{\mu}\right)$ in Coulomb gauge where the sum of the polarization tensors is $\sum_{s} \varepsilon_{\mu} \varepsilon_{\nu}=\delta_{i j}-k_{i} k_{j} / k^{2}$. The amplitude is evaluated using on shell energies where the in-medium HQ mass is determined by the many-body formalism [37], while, for reasons of gauge invariance, we restrict the gluon polarization tensor to its transverse components, i.e., $A_{T}=\left(\delta_{i j}-k_{i} k_{j} / k^{2}\right) A_{j}$ is invariant under the gauge transformation with parameter $\alpha$ as $A_{T}^{\prime}=\left(\delta_{i j}-k_{i} k_{j} / k^{2}\right)\left(A_{j}+\alpha k_{j}\right)=A_{T}$. This setup neglects other gauge invariant contributions, in particular vertex corrections 
which are, however, rather involved and beyond the scope of the present investigation. Further discussion of this point can be found in section 2.2. Note that the in-medium gluon spectral functions figuring in eq. (2.6) include a thermal mass as dictated by reproducing the QGP EoS.

Inserting the amplitude of eq. (2.10) into eq. (2.9), we have

$$
\begin{aligned}
w(\mathbf{p}, \mathbf{k}) \equiv & \frac{d N_{g}}{d t d^{3} \mathbf{k}} \\
= & \frac{1}{2 \varepsilon_{Q}(p)} \int \frac{d \nu}{(2 \pi)^{3} 2 \varepsilon_{g}(k)} \frac{d \omega^{\prime} d^{3} \mathbf{p}^{\prime}}{(2 \pi)^{3} 2 \varepsilon_{Q}\left(p^{\prime}\right)} \delta\left(\varepsilon_{Q}(p)-\omega^{\prime}-\nu\right) \delta^{(3)}\left(\mathbf{p}-\mathbf{p}^{\prime}-\mathbf{k}\right) \frac{(2 \pi)^{4}}{d_{Q}} \\
& \times 4 d_{Q} C_{F} g^{2}\left\{\left(\varepsilon_{Q}(p) \varepsilon_{Q}\left(p^{\prime}\right)-m_{Q}^{2}\right)-\frac{(\mathbf{p} \cdot \mathbf{k})\left(\mathbf{p}^{\prime} \cdot \mathbf{k}\right)}{\mathbf{k}^{2}}\right\} \\
& \times \rho_{Q}\left(\omega^{\prime}, p^{\prime}\right) \rho_{i}(\nu, k)\left[1-n_{Q}\left(\omega^{\prime}\right)\right]\left[1+n_{g}\left(\nu^{\prime}\right)\right] .
\end{aligned}
$$

In leading order for the bare $Q \rightarrow Q g$ splitting process, a restriction to on-shell energies, $\omega^{\prime}=\varepsilon_{Q}\left(p^{\prime}\right)$ and $\nu=\varepsilon_{g}(k)$ in the spectral functions leads to a vanishing rate $w(\mathbf{p}, \mathbf{k})$, since the $\delta$-functions for energy and momentum conservation cannot be simultaneously satisfied. However, at the leading dressed order (skeleton order), $w(\mathbf{p}, \mathbf{k})$ is finite since the spectral functions for outgoing heavy quark and gluons allow for off-shell energies, opening up phase space that simultaneously satisfies energy and momentum conservation. As shown in eqs. (2.6) and (2.7), the information on elastic scatterings between the outgoing partons $(Q, g)$ and the medium, and the mean free path of the outgoing partons, are encoded in the off-shell properties of the spectral functions.

Following ref. [3], the transition rate $w(\mathbf{p}, \mathbf{k})$ can be used to evaluate the drag coefficient (as used in a Langevin simulation below),

$$
A(p)=\int d^{3} \mathbf{k} w(\mathbf{p}, \mathbf{k}) \frac{\mathbf{p} \cdot \mathbf{k}}{\mathbf{p}^{2}}=\int d^{3} \mathbf{k} w(\mathbf{p}, \mathbf{k}) \frac{k_{L}}{p},
$$

where $k_{L} \equiv(\mathbf{k} \cdot \mathbf{p} / p)$ is the longitudinal momentum transfer. For high-energy (or large $k$ ) radiative processes, $k_{L} \approx k$ is a good approximation. Thus, it is useful to define the power function $k w(\mathbf{p}, \mathbf{k})$ so that the drag coefficient can be approximately expressed as $A(p) \approx \int d^{3} \mathbf{k} k w(\mathbf{p}, \mathbf{k}) / p$. If we further assume an azimuthal symmetry of the emission, we can define the power function per $k_{T}$ and $k_{L}$ phase space as

$$
W\left(p, k_{L}, k_{T}\right) \equiv k \frac{d N_{g}}{d t d k_{T} d k_{L}}=\left(2 \pi k_{T}\right) \sqrt{k_{T}^{2}+k_{L}^{2}} w\left(p, k_{T}, k_{L}\right) .
$$

The factor $2 \pi k_{T}$ originates from integrating over the azimuth angle. This power function characterizes how the radiated energy is distributed over the phase space. We can integrate over $k_{T}$ to obain

$$
x \frac{d N_{g}}{d t d x} \approx(k / p) \frac{d N_{g}}{d t d\left(k_{L} / p\right)}=\int d k_{T}\left(2 \pi k_{T}\right) \sqrt{k_{T}^{2}+k_{L}^{2}} w\left(p, k_{T}, k_{L}\right),
$$

where $\left.x=\left(k_{L}+\varepsilon_{g}(k)\right) /\left(p+\varepsilon_{Q}(p)\right)\right)\left(x \approx k_{L} / p\right.$ at high momentum) is the longitudinal momentum fraction of the emitted gluon taken from the parent heavy quark. The variable $x$ is often referred to as a light-front coordinate and commonly used in existing literature for radiative energy loss. 


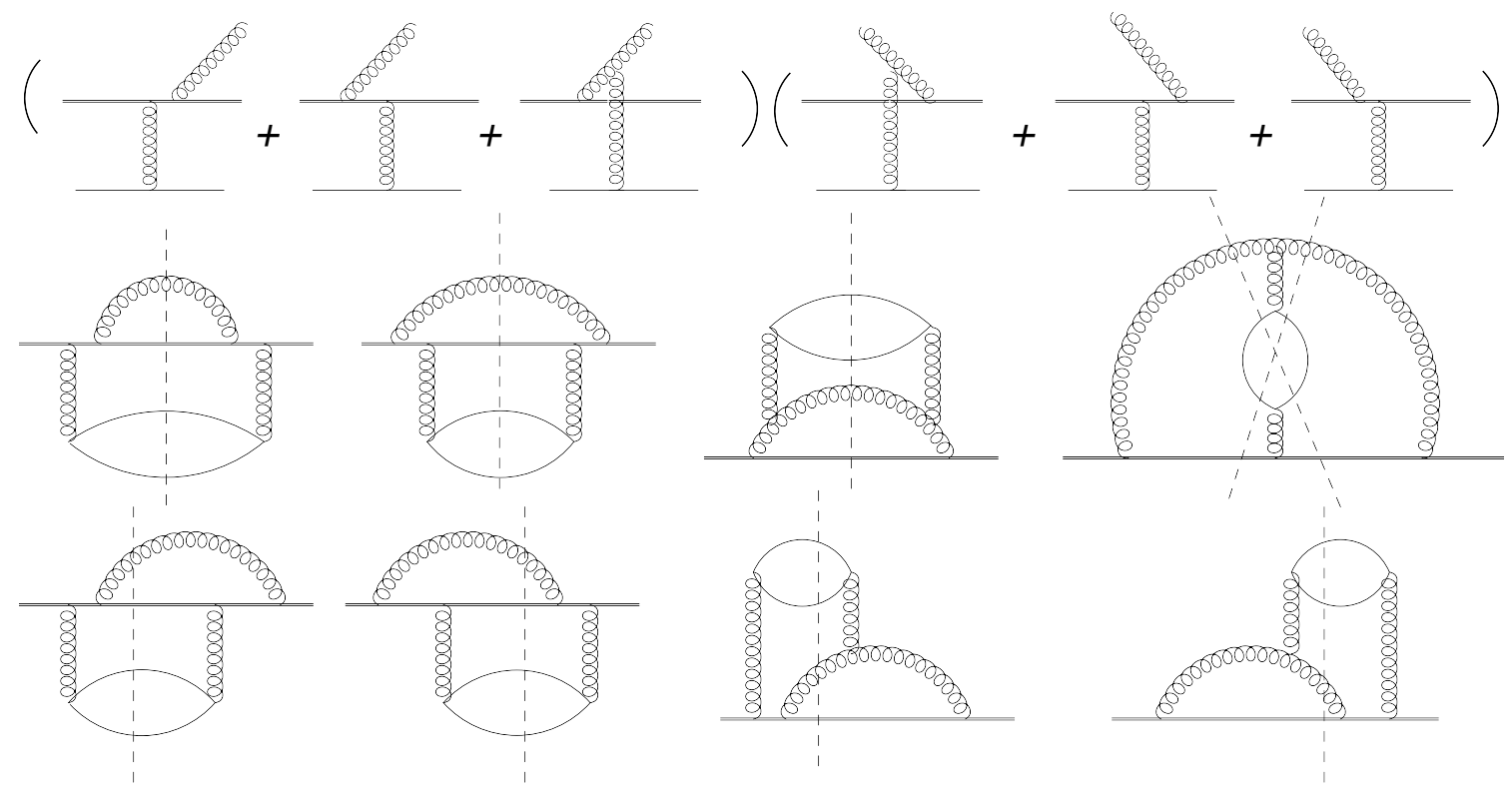

Figure 1. The first row displays the square of the Born diagrams that can be generated from the cuts of the diagrams shown in the lower two rows.

\subsection{Relation to pQCD diagrams}

To obtain a better understanding of how the NP framework employed here relates to pQCD calculations, we carry out a comparison of relevant diagrams in this section. This will also allow us to address the issue of gauge invariance for the NP case, which is a rather challenging one in the presence of resummed interactions.

We start with the tree-level pQCD diagrams $[23,38]$ shown in figure 1 . The diagrams displayed in the first row represent the (naive) order- $g^{2}$ contributions to the amplitude for two incoming quarks going into two quarks and a gluon. The sum of these amplitudes is transverse $\left(k^{\mu} M_{\mu}=0\right)$ and thus gauge invariant in the usual sense. If we contract the external legs between terms in the left and right parentheses, we obtain the selfenergy diagrams in the second and third rows, which relate to the diagrams in the first row by cutting rules (optical theorem). As discussed in the previous section, the key quantities to evaluate the radiative processes are the selfenergies, which explicitly figure in eq. (2.1) or in the spectral functions as shown in eq. (2.6). The NP selfenergies can be calculated through a series of coupled Dyson-Schwinger equations (DSEs); our approach falls into this category. The structure of the equations is controlled by the skeleton diagram expansion where an example is shown in figure 2. In this example, by inserting the dressed vertex (first row) and the dressed gluon (second row) into the equation in third row, we can generate the pQCD selfenergy diagrams shown in second and third rows in figure 1. With a specific choice of the skeleton expansion, the diagrams generated by DSEs can encompass any finite-order perturbative diagrams. In other words, using the DSEs perturbatively is an alternative and equivalent diagram expansion method for the perturbative diagram expansion. With a specific truncation scheme, the DSEs form a closed system of equations that allow NP 

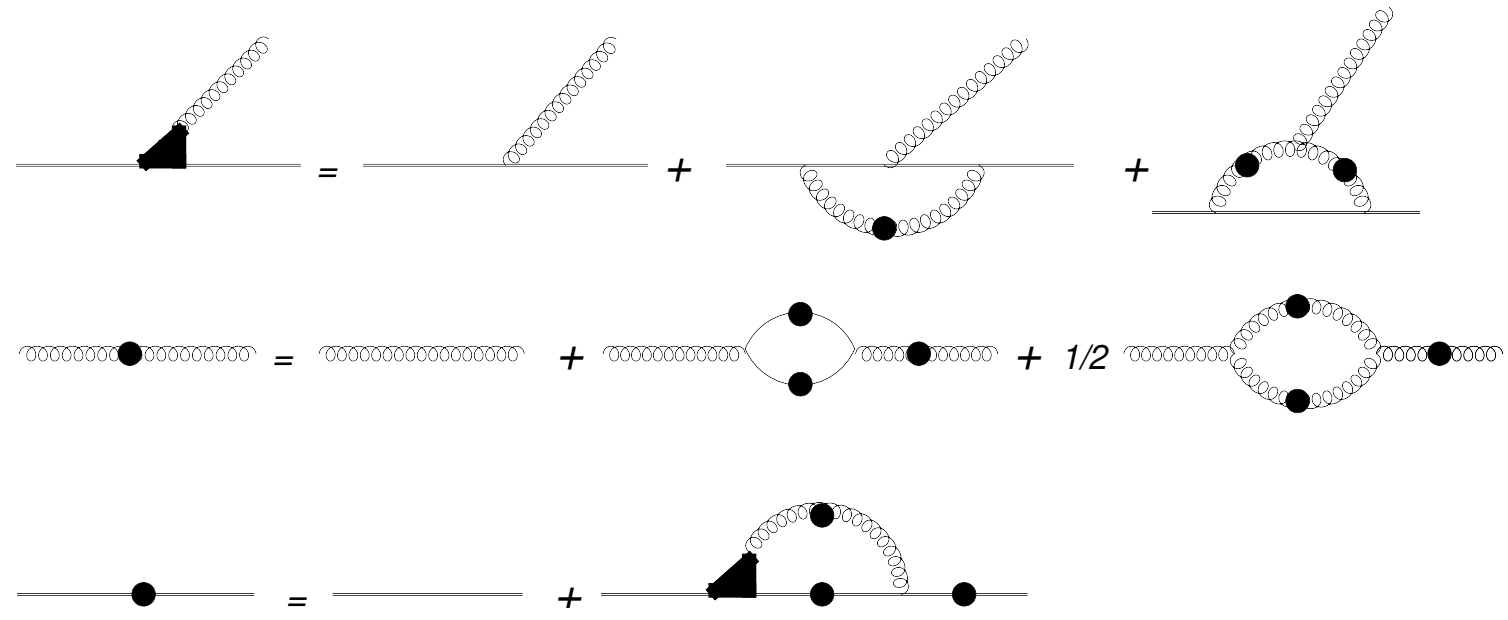

Figure 2. The coupled set of DSEs with skeleton diagrams that can generate the pQCD diagrams in figure 1. The dots on the lines indicate dressed propagators.

solutions. These solutions can be obtained through selfconsistent iterations. They resum the diagrams with specific patterns (such as ladder diagrams, rainbow diagrams and ring diagrams) to infinite order, which goes beyond any fixed-order perturbative expansion and thus can provide meaningful results at large coupling strength. However, these truncation schemes usually compromise explicit gauge invariance since they select a particular subset of diagrams to resum. There is no general solution to this problem yet in the strongly interacting regime, although progress has been made in resolving this issue in vacuum [39-41].

In the present article we focus on NP features at large interaction strength. We compromise on exact gauge invariance by carrying out the $t$-channel ladder resummation but restrict ourselves to projecting out the gauge invariant part by applying the transverse projector, $M_{T}^{\mu}=P_{T}^{\mu \nu} M_{\nu}$, ${ }^{1}$ i.e., we only keep the bare radiation vertex with emission of the gauge invariant transverse mode, while in principle also a gauge invariant longitudinal mode is available in medium $[42,43]$. Thereby we also neglect the vertex corrections which would be required in connection with an in-medium emission vertex. More rigorous methods are beyond the scope of this work.

To highlight model-independent information obtained from our apporach, we conduct calculations for four setups with different inputs representing different NP effects and compare their differences. Since they are all calculated in one model, potential gauge artifacts are expected to be similar in the different setups so that the differences between them give insights into model-independent features.

\subsection{Four cases of NP effects}

Within the formalism laid out above the NP effects that we study in this work are essentially encoded in the spectral functions (selfenergies) of eq. (2.7) which figure in the emission rate, eq. (2.11). These effects are mainly from 3 sources: confining interactions, ladder

$$
{ }^{1} P_{T}^{i j}=\delta^{i j}-k^{i} k^{j} / k^{2}, P_{T}^{0 \mu}=P_{T}^{\mu 0}=0[42] .
$$




\begin{tabular}{|c|c|c|c|c|}
\hline Cases & (1) $V_{C}$ Born & $(2) V_{C S}$ Born & $(3)$ T-matrix Onshell & (4) T-matrix Offshell \\
\hline Interaction & Coulomb & Coulomb+String & Coulomb+String & Coulomb+String \\
\hline Resummation & 2nd-order & 2nd-order & All order & All order \\
\hline Medium & quasi-particle & quasi-particle & quasi-particle & off-shell spectra \\
\hline
\end{tabular}

Table 1. Four cases are labeled by numbers or abbreviations in the first row where their differences in interaction, resummation scheme, and medium content are listed in the second, third, and fourth rows respectively.
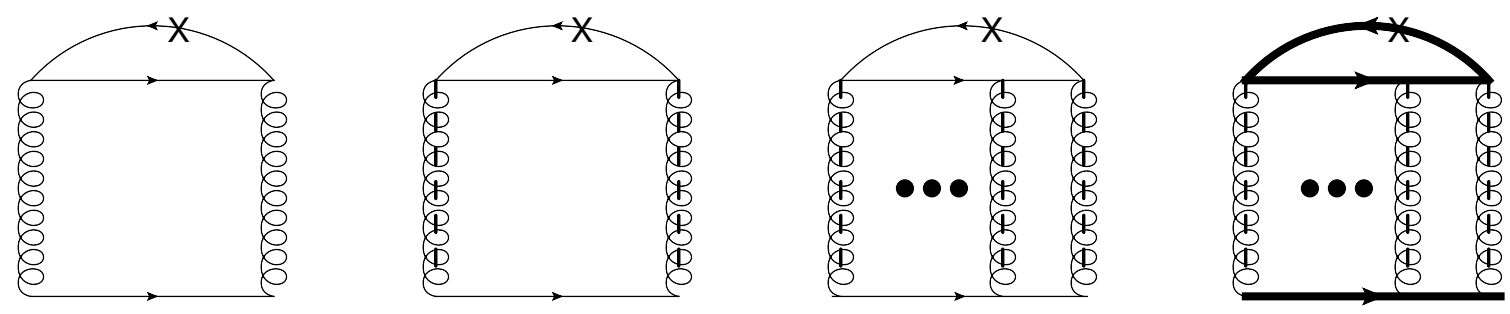

Figure 3. Self-energy diagrams for the four different cases discussed in table 1. Helical lines denote $V_{C}$, helical lines with double dashed lines denote $V_{C S}$, thin solid lines with arrows denote on-shell quasi-particle partons $(q, g, \mathrm{HQ})$, thick solid lines with arrows denote off-shell dressed partons.

resummations, and off-shell interactions, each of them playing a critical role in our manybody approach to heavy quarks in the QGP [36]. In the following we will define 3 scenarios, in addition to the full results, which allow us to exhibit their effects on gluon radiation from a heavy quark traveling through the QGP.

The four cases are summarized in table 1, illustrated in figure 3 and defined in detail in the following.

Case-1, " $V_{C}$ Born", contains none of the three NP effects mentioned above, and as such represents the perturbative baseline in our approach. It only includes color-Coulomb interactions with coupling constant and Debye mass taken from the strongly coupled solution (SCS) in ref. [37]. It does not contain a $t$-channel ladder resummation, and we only keep the second-order Born diagrams as shown in figure 3, which are the leading-order diagrams that generate an imaginary part of the selfenergy and consequently a finite width of the spectral functions of the outgoing heavy quark and gluon in eq. (2.11) as required for a finite radiation rate. These diagrams are related to the squared Born scattering amplitude using the cutting rule. The thermal-medium partons are taken as zero-width quasiparticles with their masses fitted to the QGP EoS as discussed in our previous work [37]. This case is most closely related to the treatment in typical pQCD calculations, although some differences persist, most notably the large thermal masses of the emitted gluon.

Case-2, " $V_{C S}$ Born" adds a NP effect to case-1, namely the confining interaction (potential) again with parameters taken from the SCS of ref. [37], illustrated in the second panel of figure 3 by the extra bars in the gluon exchange lines. This is still done in leadingorder Born approximation and with the same medium as in case-1.

Case-3, "T-matrix Onshell", additionally includes the $t$-channel ladder resummation in the heavy-light and light-light $T$-matrices (used to compute the outgoing HQ and gluon 
selfenergies) compared to case-2, which is illustrated in the third panel of figure 3 . The other components are the same as in case (2).

Finally, case-4, "T-matrix Offshell", uses finite width off-shell spectral functions for the thermal-medium partons. As shown in the last panel of figure 3, we now dress all internal lines in a selfconsistent calculation of selfenergies and $T$-matrices. In addition to the medium partons in the upper part of the diagram, the incoming and internal HQ lines are also dressed. The off-shell spectral functions are taken from the full solution of the SCS as discussed in ref. [36]. Case-4 is the most complete and consistent many-body theory calculation for the rate within our current approach, containing all three NP effects as highlighted at the beginning of this section.

Let us briefly reiterate on the role of the thermal-medium partons in the HQ transport calculation. For cases-1, -2 and -3 , we use a zero-width quasiparticle medium. For case-4, the broad spectral functions that represent the off-shell medium are the predictions of the SCS in ref. [37]. Both scenarios describe the EoS of the QGP, implying that the effective density of "scattering centers" is quite similar, in order to focus on the genuine effects of the NP ingredients. Also note that the resummations carried out in the present work refer to $t$-channel ladder diagrams for a single (dynamic) scattering center. Higher orders due to multiple scattering centers and multiple gluon emission are implicitly included in the Langevin simulation, which is, of course, incoherent and thus neglects the Landau-Pomeranchuk-Migdal (LPM) effect [44-46]. While the latter is essential for radiation off light-flavor partons, it is mass suppressed for heavy quarks due to reduced formation times. A rigorous implementation of coherence effects in transport approaches remains challenging, see, e.g., ref. [25] for an approximate treatment in the HQ context.

\section{$3 \quad$ Numerical analysis}

In this section, we present and analyze the numerical results for the four cases defined above. We first study various microscopic quantities and then discuss how they manifest themselves in transport coefficients. We start by presenting the pertinent spectral functions for heavy quarks and gluons in section 3.1, followed by the corresponding power spectra in section 3.2. The resulting drag coefficients and fractional energy loss are calculated in section 3.3. In section 3.4 we discuss our results in comparison to a pQCD calculation from the literature.

\subsection{Spectral properties of radiated partons}

The spectral functions of the outgoing heavy quark and gluon are the key quantities for calculating the rate of radiation for the $Q \rightarrow Q g$ process given by eq. (2.11). These spectral functions are shown in figure 4 for our 4 different cases, each one for three HQ and gluon momenta, $p=2,10,40 \mathrm{GeV}$ and two temperatures, $T=0.194,0.4 \mathrm{GeV}$, as a function of energy around their on-shell values. We recall that for cases 1-3, these spectral functions differ from those in the internal lines in figure 3 where they are zero-width quasiparticles. For all cases, a markedly different feature from the standard pQCD approach are the rather large thermal-parton masses dictated by the constraints from the QGP EoS and microscopically related to the Polyakov loop encoding the nontrivial information of the confinement [36] 
generated selfconsistently from the confining potential. Similar features from including effects of the Polyakov loop have recently been found from a different perspective, within the Polyakov-quark-meson model [47]. With a gluon mass of around $1 \mathrm{GeV}$ at low temperature, soft radiation is heavily suppressed. With increasing 3-momentum at a fixed temperature (from top to bottom row in figure 4), the spectral functions in the " $V_{C^{-}}$Born" case (left panels) show an increasing width while the opposite trend is found for the other 3 cases which is caused by the presence of the confining interaction whose strength rapidly decreases with increasing momentum (transfer), especially at low temperature. At the same time, at high momentum (bottom row), there is little difference among the different cases, with well defined quasiparticles characteristic for pQCD calculations. These features are a direct reflection of our previous findings that the QGP medium at moderate temperatures is strongly coupled at large wavelengths while recovering a more weakly interacting quasiparticle structure with increasing resolution [36]. For the temperature dependence at a fixed 3-momentum, the " $V_{C}$ Born" case shows a clear increase in width from low to high temperatures, which is a consequence of the increasing thermal-parton density which overwhelms the moderate loss in interaction strength caused by a stronger Debye screening. This remains true for the other three cases at high momenta, while at low momentum this trend is much less pronounced, even slightly inverted for the "T-matrix Offshell" case. At the lowest temperature and momentum $(T=0.194 \mathrm{GeV}, p=2 \mathrm{GeV})$, all 3 cases $(2-4)$ involving the confining interaction show appreciable distortions from a simple Lorentzian shape caused by a collective mode that can even generate an additional peak that becomes visible due to the much increased interaction strength and the broad original quasi-particle peak which is pushed to somewhat larger energies due to the level repulsion [35, 36] (in the " $V_{C}$ Born" case one only finds a slight distortion from the Lorentzian shape). In particular, the gluon spectral functions exhibit substantial strength, even collective modes, in the spacelike region, i.e., for $\omega<p$ (and for both temperatures), which is the most relevant one for the emission process off an incoming on-shell heavy quark. The spacelike strength tends to increase with the inclusion of more NP effects. At high temperature and low momentum $(T=0.400 \mathrm{GeV}, p=2 \mathrm{GeV})$, the inclusion of the string interaction alone, at the Born level, strongly enhances the strength in the space-like region, compare the dash-dotted blue lines in the 2nd and 1st panel of the upper row in figure 4. Although the addition of resummation (case-3) and off-shell (case-4) effects further distorts the spectral functions (cf. 3rd and 4th panel), they do not lead to significant differences for the phase space in the spacelike region. For higher momentum, $p=10 \mathrm{GeV}$, at both temperatures, the change of the spectral properties by adding the NP effects is less pronounced. Still, the largest modification is generated by the inclusion of the string interaction at low temperatures, while the further differences in cases 3 and 4 are not significant. At high temperature and $p=10 \mathrm{GeV}$, even the inclusion of the string interaction is no longer significant.

\subsection{Power spectra}

Inserting the spectral functions discussed in the previous section into the rate, eq. (2.11), we can evaluate the power spectra of radiation defined in eqs. (2.13) and (2.14). The former essentially corresponds the radiation spectrum, while the latter, with the transverse 

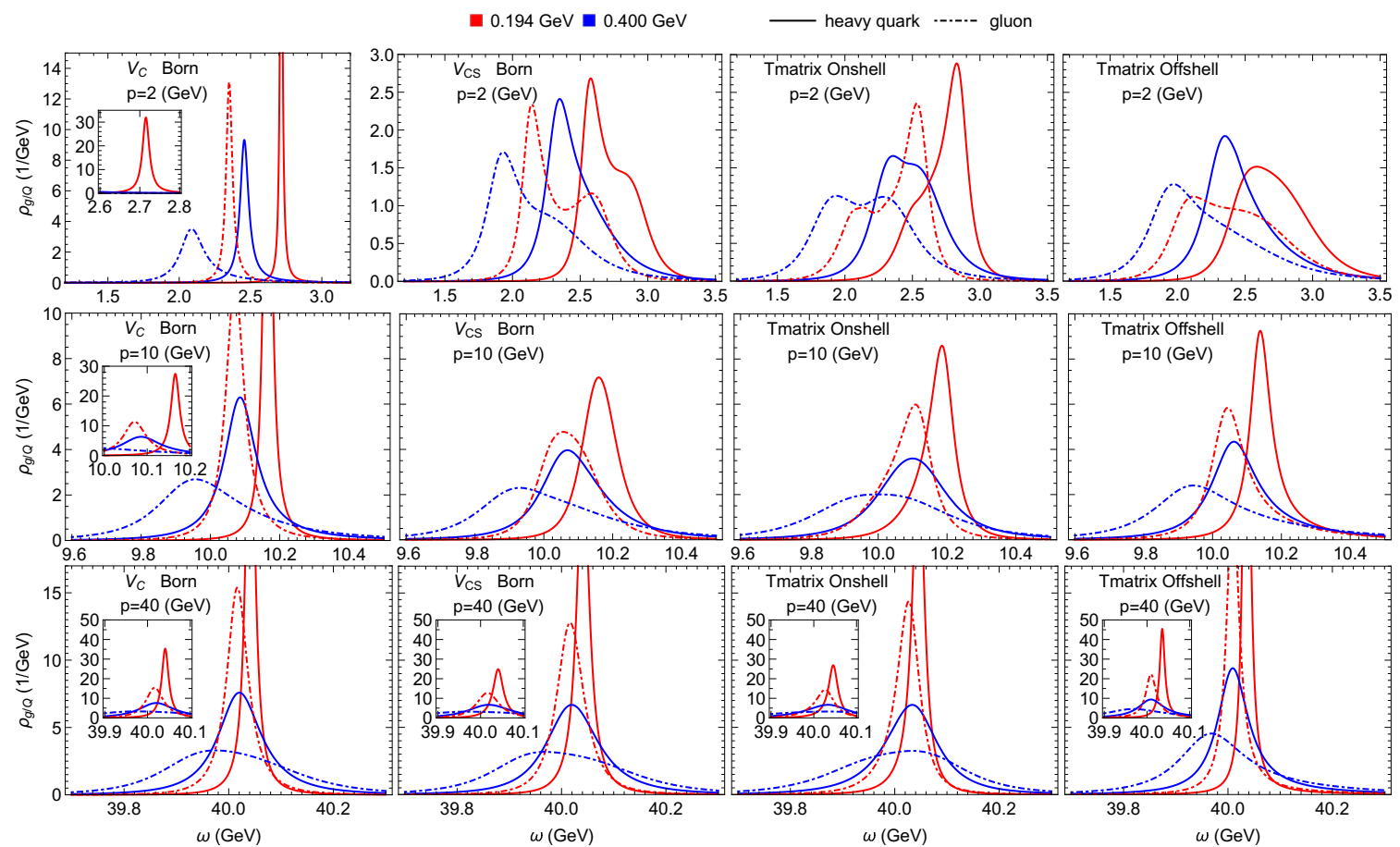

Figure 4. Spectral functions of charm quarks (solid lines) and gluons (dash-dotted lines) for cases 1 through 4 from left to right, with different temperatures and momenta.

momentum transfer integrated, is more readily interpreted as the radiative energy loss of the heavy quark. At the end of this section we compute the transverse-momentum broadening coefficient, $\hat{q}$, for the " $V_{C}$ Born" and " $V_{C S}$ Born" cases and illustrate how they relate back to their differences in the power spectra.

In figure 5 we display contour plots of the power spectra in the $k_{T}-k_{L}$ plane, where each of the 4 interaction cases is represented by each of the four columns, and each row represents a pair of temperature and HQ 3-momentum, for $p=10 \mathrm{GeV}$ with $T=0.194,0.4 \mathrm{GeV}$ in the upper 2 rows, and $p=40 \mathrm{GeV}$ with $T=0.194,0.4 \mathrm{GeV}$ in the lower 2 rows. The power spectra are projected onto the $k_{L}$ values (i.e., integrated over $k_{T}$ ) and shown in figure 6 , where the 4 combinations of $p$ and $T$ are combined into a single plot for each of our 4 cases. The generic features of the power spectra are a suppression of very soft radiation, i.e., for small $k_{L}$, due to the large effective gluon mass, and a suppression of the collinear radiation, i.e., for small angles, $k_{T} / k_{L}$, which is the well known deadcone effect [48]. As expected, the radiation power increases with both temperature and momentum, roughly by one order of magnitude when going from $T=0.194$ to $0.4 \mathrm{GeV}$, and between a factor of 5 (for case- 1 at $\mathrm{T}=0.194 \mathrm{GeV}$ ) and $10 \%$ (for cases $2-4$ at $T=0.4 \mathrm{GeV}$ ) when going from $p=10 \mathrm{GeV}$ to $40 \mathrm{GeV}$ (note that the contour plots have been scaled to their respective maxima in each panel, as quoted in the figure legend). The $k_{T}$ distributions of the power spectra tend to narrow down with increasing $p$.

The most signficant of the NP effects is the inclusion of the string interaction, when going from case- 1 to case- 2 , and it is more pronounced for lower temperatures, as borne 


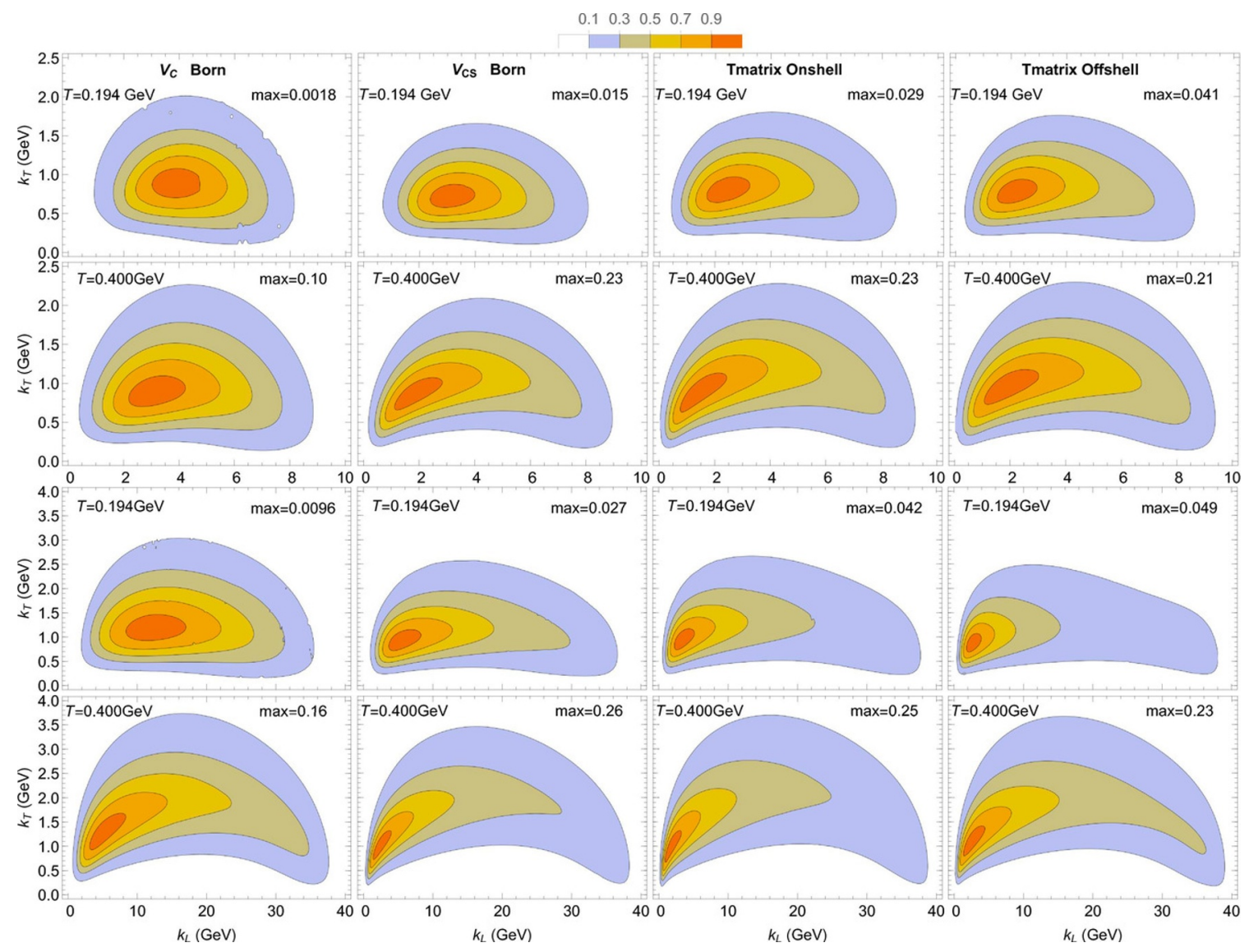

Figure 5. Contour plots of the normalized radiation spectrum, $W\left(k_{T}, k_{L} ; p\right) / W_{\max }$ in the plane of longitudinal $\left(k_{L}\right)$ and transverse momentum $\left(k_{T}\right)$ of the emitted gluon for fixed HQ momentum and temperature. Each column corresponds to one of the four cases of NP effects, while the upper (lower) two rows are for $p=10(40) \mathrm{GeV}$, and for different temperatures $(T=0.194(0.4) \mathrm{GeV}$ for rows 1 and $3(2$ and 4$)$ ). The maximum values, $W_{\max }$, of each the power spectra are labeled as "max" in units of $\mathrm{GeV} / \mathrm{fm}$ in the plots.

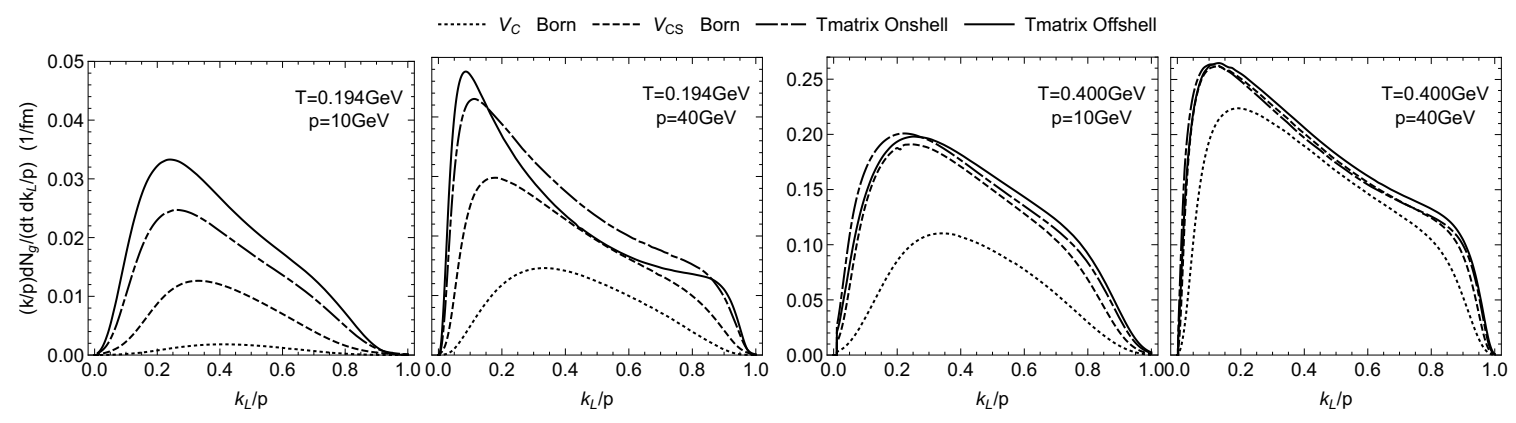

Figure 6. The $k_{T}$-integrated power spectra, $\frac{(k / p) d N}{d t d\left(k_{L} / p\right)} \approx \frac{x d N}{d t d x}$, as a function of longitudinalmomentum fraction $\left(k_{L}\right)$ of the emitted gluon for the four different cases in each figure, at low (high) temperature in the left (right) two panels, each for two different HQ momenta. 

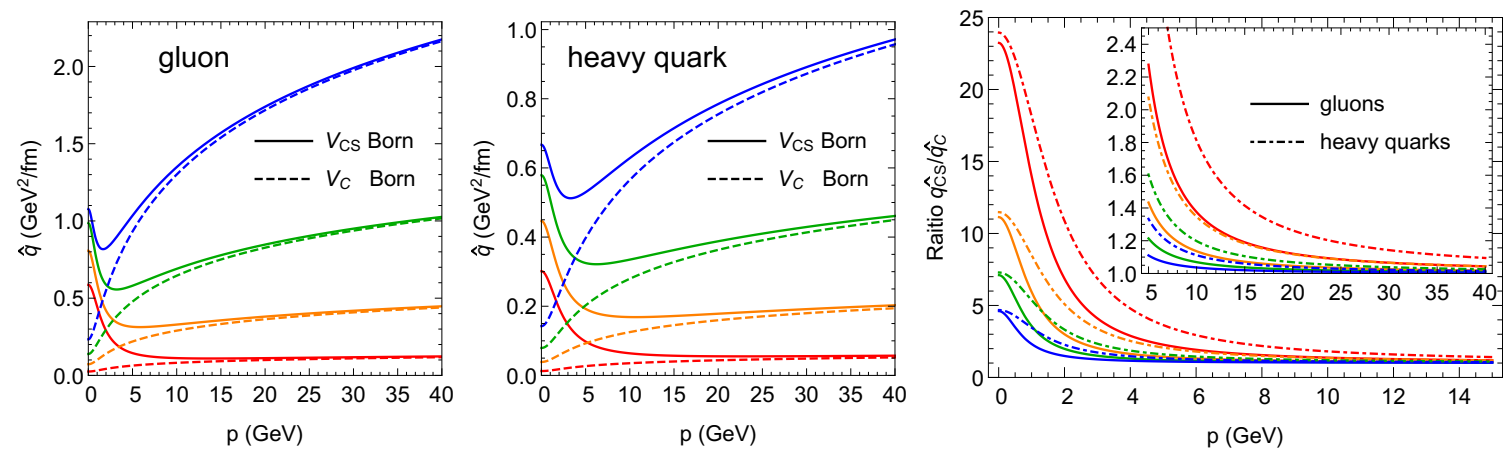

Figure 7. The transverse-momentum broadening coefficient, $\hat{q}$, of a gluon (left panel) and a charm quark (middle panel), calculated with a fixed coupling constant for different temperatures and HQ momenta in cases 1 (dashed lines) and 2 (solid lines). The right panel shows the ratios of the solid over dashed curves from the left and middle panel (solid curves for gluons and dashed curves for charm quarks). In all panels the red, orange, green and blue curves represent temperatures $T=0.194,0.258,0.32$ and $0.4 \mathrm{GeV}$, respectively.

out from the maximum values in the power spectra which increase between a factor of $\sim 30$ for $(p, T)=(10,0.194) \mathrm{GeV}$ and factor of $\sim 1.6$ for $(p, T)=(40,0.4) \mathrm{GeV}$. However, at low temperature, the additional NP effects in cases 3 and 4 (resummation and off-shell effects) are still rather significant, especially for rather soft gluons $(x \lesssim 0.2)$, see the first and second panel in figure 6 , while they have a small impact at high $T$, cf. third and fourth panel in figure 6 . These features directly reflect the discussion of the spectral properties of the outgoing gluon and heavy quark in the previous section.

The high-energy transport parameter $\hat{q}$ represents the average transverse-momentum transfer per mean-free-path of the heavy quark. It has been related to the elastic part of the commonly calculated coefficients in a Fokker-Planck equation [3], specifically the transverse-momentum diffusion coefficient, $B_{0}^{\mathrm{el}}$, as $\hat{q}=4 B_{0}^{\mathrm{el}} \approx 4 T \epsilon_{Q}(p) A^{\mathrm{el}}(p)[49]$; the second approximate equality involving the elastic friction coefficient, $A^{\text {el }}$, follows from the Einstein relation (which is routinely enforced in practical applications). In pQCD based approaches [31], $\hat{q}$ is usually factorized and used as a fit parameter encoding the NP properties of the QGP. Here we can check whether this factorization also holds at a more differential level, e.g., for the power spectra. Toward this end, we first compute the $\hat{q}$ for the " $V_{C}$ Born" and " $V_{C S}$ Born" scenarios from $B_{0}^{\text {el }}$ with our Born amplitudes, to illustrate how the string interaction affects this widely used jet transport coefficient. The results are shown in figure 7 . We here use a fixed coupling constant which implies that $\hat{q}$ increases logarithmically at high momentum, which is similar to the behavior found in ref. [50] but different from results evaluated with a running coupling which typically come out approximately momentum independent. As expected from our preceding discussion of spectral functions and power spectra, the results for " $V_{C S}$ Born" and " $V_{C}$ Born" converge toward each other at high momentum, but the former is significantly enhanced for momenta below about $10 \mathrm{GeV}$. This scale can thus be identified as the transition regime from perturbative 


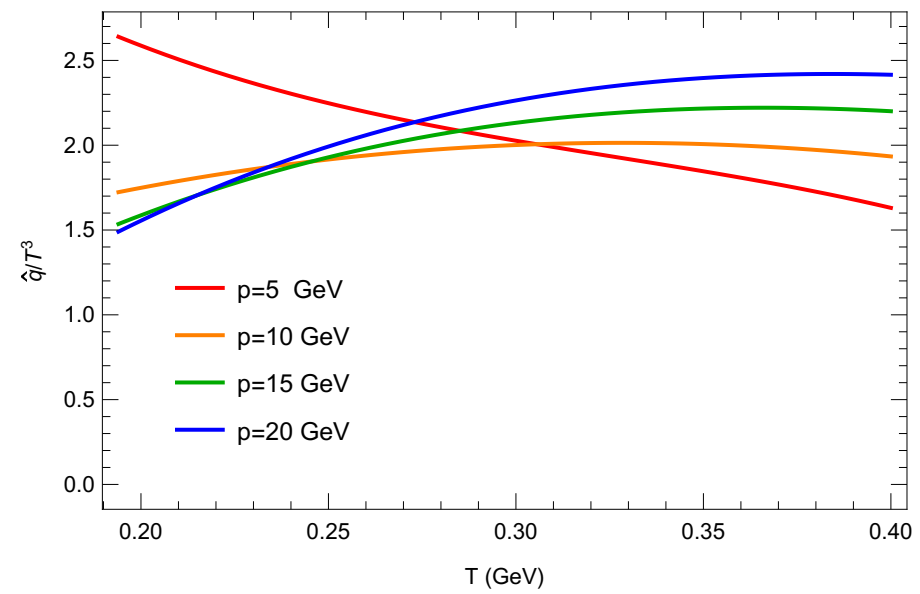

Figure 8. Dimensionless-scaled HQ transport coefficient, $\hat{q} / T^{3}$, in the " $V_{C S}$ Born" scenario as a function of temperature for different HQ momenta.
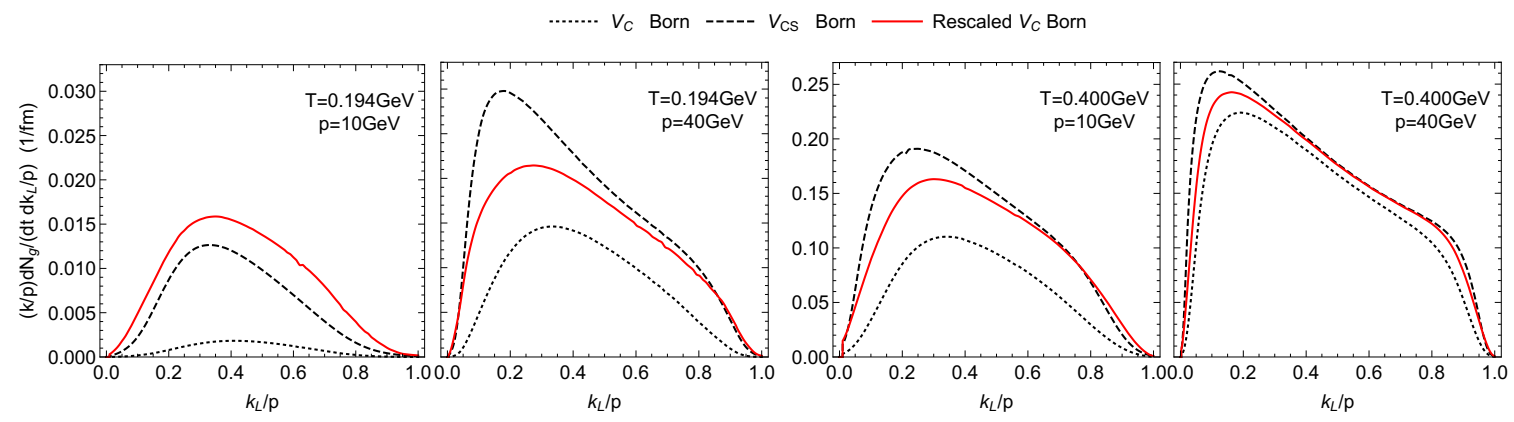

Figure 9. Comparison of the $k_{T}$-integrated power spectra, $\frac{(k / p) d N}{d t d\left(k_{L} / p\right)} \approx \frac{x d N}{d t d x}$, as a function of longitudinal-momentum fraction $\left(k_{L}\right)$ of the emitted gluon for the " $V_{C}$ Born" case (dotted lines), the " $V_{C S}$ Born" case (dashed lines), and the " $V_{C}$ Born" case augmented with the $\hat{q}$ scaled enhancement factor from the two cases as defined in eq. (3.1) (solid lines), at low (high) temperature in the left (right) two panels, each for two different HQ momenta.

to nonperturbative. A NP enhancement factor, defined in terms of the ratio of the $\hat{q}$ 's from the 2 scenarios, is shown in the right panel of figure 7 ; while the absolute value of the gluon $\hat{q}$ is larger than the one for charm quarks by about a factor of 2 , the relative NP enhancement factor is actually larger for heavy quarks than for gluons. The temperature dependence of the HQ $\hat{q}$, scaled by $T^{3}$, is displayed in figure 8 for the " $V_{C S}$ Born" case; it reiterates the importance of the string interaction at relatively low momenta, significantly enhancing the coupling strength toward small temperatures.

We can the test the "factorization hypothesis" referred to above by multiplying the NP enhancement factor into the power spectra of " $V_{C}$ Born" and compare it to the power spectra of " $V_{C S}$ Born". More concretely, we define the "rescaled $V_{C}$ Born" power spectrum as

$$
\left[\frac{(k / p) d N_{g}}{d t d\left(k_{L} / p\right)}\right]_{\text {scaled }}=\frac{(k / p) d N_{g}}{d t d\left(k_{L} / p\right)} \frac{\hat{q}_{g}^{C S}\left(k_{L}\right)}{\hat{q}_{g}^{C}\left(k_{L}\right)} \frac{\hat{q}_{Q}^{C S}\left(p-k_{L}\right)}{\hat{q}_{Q}^{C}\left(p-k_{L}\right)},
$$




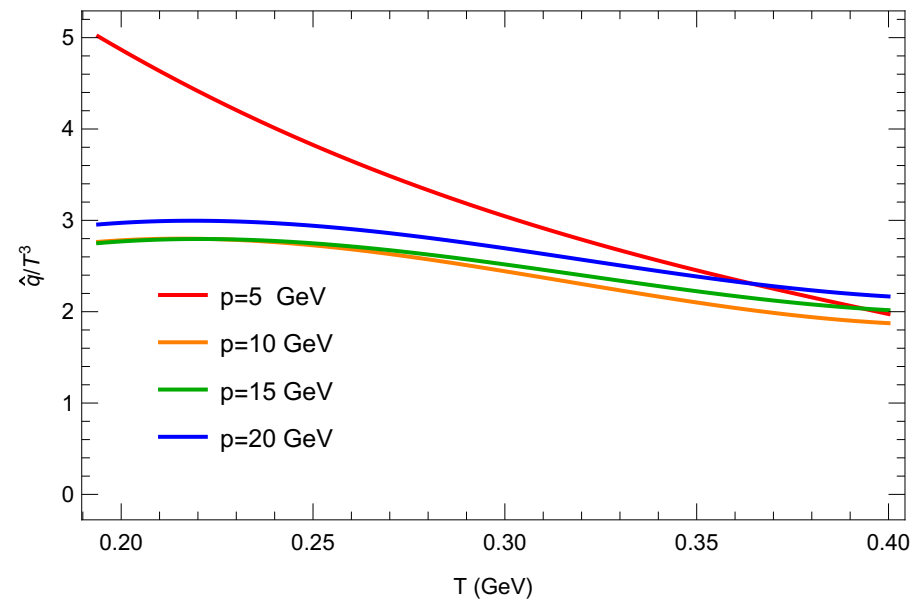

Figure 10. Dimensionless-scaled HQ transport coefficient, $\hat{q} / T^{3}$, in the " $T$-matrix Offshell" scenario as a function of temperature for different $\mathrm{HQ}$ momenta.

which accounts for the NP enhancement in both the emitted gluon and the outgoing heavy quark at their respective momenta. The results, plotted in figure 9, show that this prescription indeed gives an approximate mapping from " $V_{C}$ Born" to " $V_{C S}$ Born", although deviations of up to $\pm 30 \%$ or so persist, especially at low temperature.

For completeness, we display in figure 10 our full result for the temperature dependence of $\hat{q}$, i.e., in the " $T$-matrix Offshell" scenario, as obtained from the elastic friction coefficient, $A^{\mathrm{el}}(p)$, by use of the Einstein relation based on figure 11 of ref. [37] (which involves a partial-wave expansions up to $l=8$ to achieve a decent convergence at high momentum). Its magnitude is further enhanced by close to a factor of 2 at low momenta compared to the " $V_{C S}$ Born" scenario, while a significant temperature dependence mostly arises for momenta below $10 \mathrm{GeV}$ and temperatures below $\sim 300 \mathrm{MeV}$.

\subsection{Drag coefficient and energy loss}

The drag (or friction) coefficient, $A(p)$, is essentially an integral of the power spectrum over $k_{T}$ and $k_{L}$, divided by the $\mathrm{HQ}$ momentum, $p$. It quantifies the fractional energy loss per unit time. Since for high-energy quarks the velocity is near the speed of light, it can be regarded as the fractional energy loss per unit path length. In the following, we discuss how the different cases affect the radiative drag coefficient, and implement them into a Langevin simulation for a charm quark in the background of QGP of finite size and fixed temperature, sometimes referred to as a "QGP brick". Several different energy loss approaches for charm and bottom quarks have been compared in this setup in ref. [32].

In the first row of figure 11, we show the drag coefficient as a function of HQ momentum for the four different cases in each panel for different temperatures. At large momentum $(p=40 \mathrm{GeV})$, the drag coefficients essentially saturate at high $p$ and show a close-to-linear increase with temperature over the considered range, $T=0.194-0.4 \mathrm{GeV}$, in all four cases; the latter is still true at lower momentum, $p=10 \mathrm{GeV}$, except for the " $V_{C}$ Born" case which is closer to a quadratic temperature dependence. The drag coefficient is strongly 

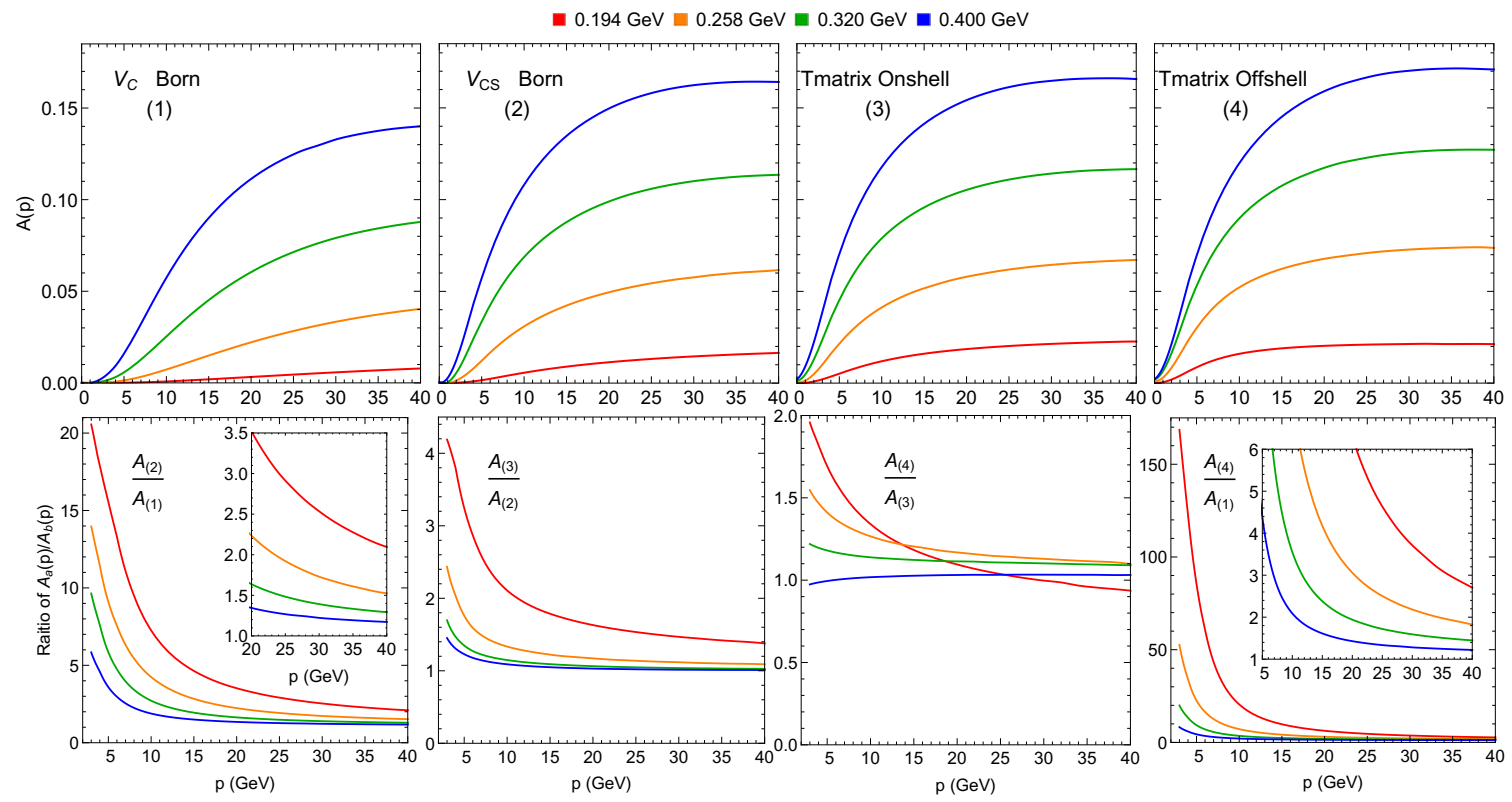

Figure 11. The first row shows the drag coefficients for the four cases of NP effects, as a function of momentum for 4 different temperatures each. In the second row the first three panels show the ratios $A_{(i+1)} / A_{i}$ between the subsequent drag coefficients of the four cases, with the last panel showing the total effect, $A_{4} / A_{1}$.

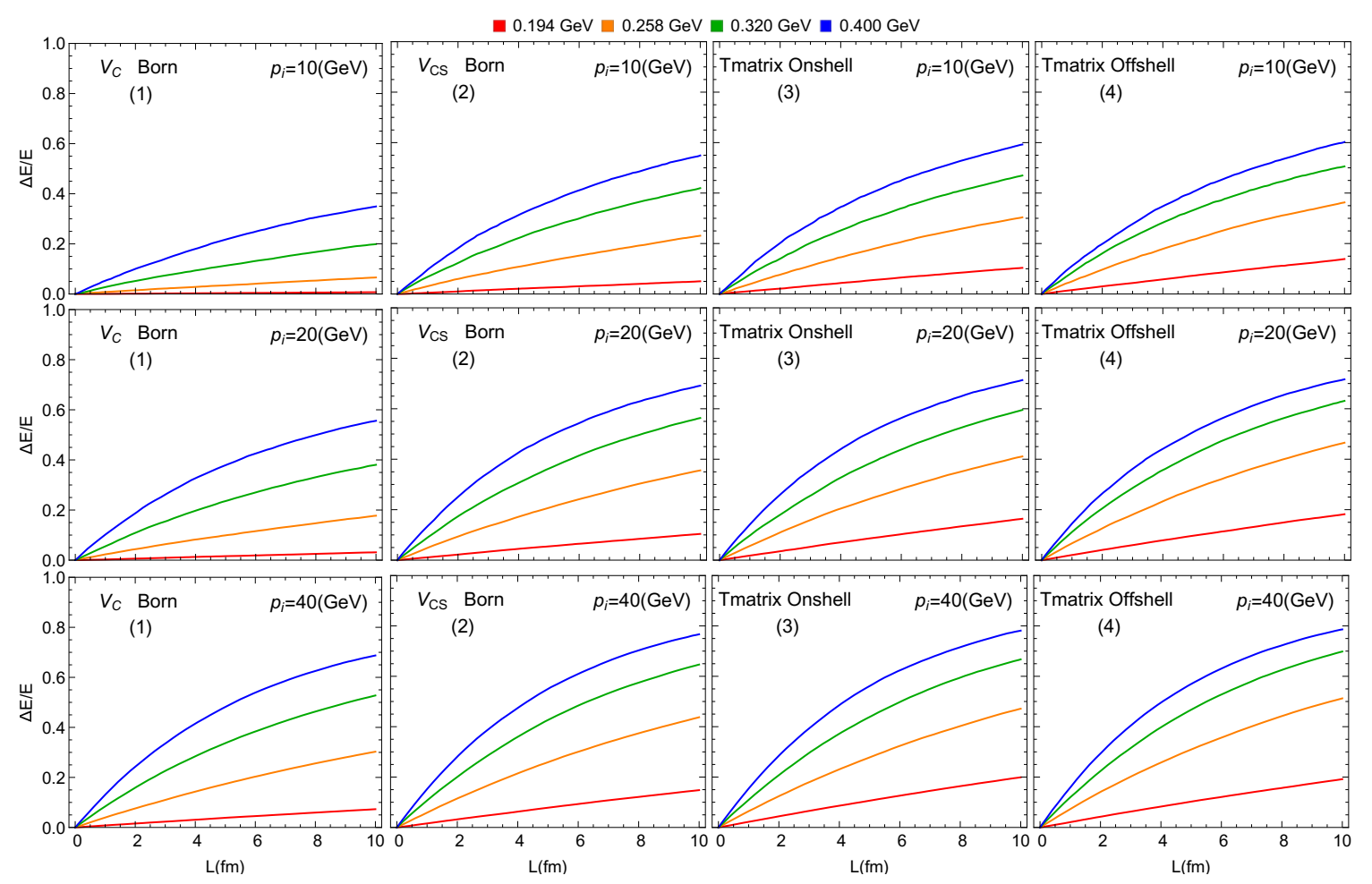

Figure 12. The fractional energy loss, $\Delta E / E=\left(\varepsilon_{Q}(0)-\varepsilon_{Q}(L)\right) / \varepsilon_{Q}(0)$, for the 4 different cases of NP effects, for initial HQ momenta of $p_{i}=10,20,40 \mathrm{GeV}$ in the upper, middle and bottom row, respectively, and for 4 temperatures in each panel. 
suppressed at small momentum, as a consequence of the large thermal-gluon mass. To better quantify the enhancement with the subsequent inclusion of NP effects we plot in the second row of figure 11 the ratio between adjacent cases. The ratio $A_{(2)} / A_{(1)}$ in the first panel, " $V_{C S}$ Born" relative to " $V_{C}$ Born", shows the largest relative enhancement, most pronounced at lot $T$ and low $p$, as seen before, reiterating the importance of the string interaction, even at the Born level. The ratio $A_{(3)} / A_{(2)}$ in the second panel exhibits a more moderate, but still substantial enhancement due to the resummation of $t$-channel ladder diagrams in the heavy-light $T$-matrix. The ratio $A_{(4)} / A_{(3)}$ in the third panel indicates that the additional off-shell treatment of the spectral function of the medium partons has a still smaller impact; at high momentum, even the temperature hierarchy of this effect is not definite. The combined impact of the NP effects on our pQCD baseline scenario, quantified by the ratio $A_{(4)} / A_{(1)}$ shown in the lower right panel of figure 11, enhances the radiative contribution to the drag coefficient by up to a factor of $\sim 150$ at small momenta, below $3 \mathrm{GeV}$, and at temperatures close to $T_{\mathrm{pc}}$. This is, however, somewhat academic, since its absolute magnitude is very small compared to the elastic contribution in the same framework [37], e.g., $A_{\mathrm{el}}(p=3 \mathrm{GeV}, T=0.194 \mathrm{GeV})=0.1 / \mathrm{fm}$ compared to $0.003 / \mathrm{fm}$ for the radiative drag (after the NP enhancement). On the other hand, already at $p=5 \mathrm{GeV}$ and $T=0.4 \mathrm{GeV}$, where both contributions are near $0.08 / \mathrm{fm}$, the NP enhancement of a factor of $\sim 4$ is certainly relevant.

Finally, we implement the radiative transport coefficients into a Langevin simulation given by incremental time steps for the HQ position and momentum,

$$
d \mathbf{x}=\frac{\mathbf{p}}{\varepsilon_{c}(p)} d t, \quad d \mathbf{p}=-\Gamma(p) \mathbf{p} d t+\sqrt{2 d t D(p)} \boldsymbol{\rho},
$$

where $\boldsymbol{\rho}$ is a random number determined from a Gaussian distribution function, $P(\boldsymbol{\rho})=$ $(2 \pi)^{-3 / 2} e^{-\boldsymbol{\rho} / 2}$, and the transport coefficients are

$$
\Gamma(p)=A(p), \quad D(p)=B_{0}(p)=B_{1}(p)=\int_{p}^{\infty} d q q \Gamma(q) e^{-\frac{\varepsilon_{Q}(q)-\varepsilon_{Q}(p)}{T}} \approx \Gamma(p) \varepsilon_{Q}(p) T .
$$

We use the pre-point Ito setup in which the relaxation rate is equal to the drag coefficient, and the momentum diffusion coefficients are defined via the Einstein relation and obtained by solving the differential equation $\Gamma(p)=1 / \varepsilon_{Q}\left(D(p) / T-d D(p) / d \varepsilon_{Q}\right)$ with the boundary condition $D(\infty)>0$. In figure 12 , we show the results of 3D Langevin simulations for a charm quark inside a static QGP brick with a $10 \mathrm{fm}$ path length. We have verified that the fluctuation term in eq. (3.2) is not important for the average energy loss and therefore the Langevin equation can be replaced by a 1-D differential equation, $d p=-\gamma(p) \varepsilon_{Q}(p) d x$. Without the LPM effect [46], the energy loss for small path lengths is near linear. For large path lengths, the negative curvature is caused by the fact that the radiative drag coefficient decreases with decreasing HQ momentum. At low temperature and low momentum, our many-body approach generally predicts a much smaller radiation and energy loss than pQCD-based approaches (cf. figure 27 in ref. [32]). The main reason is the large gluon mass creating a large energy threshold for the emission. However, the thermal masses are a key feature of our many-body approach, essentially constrained by the QGP equation of 


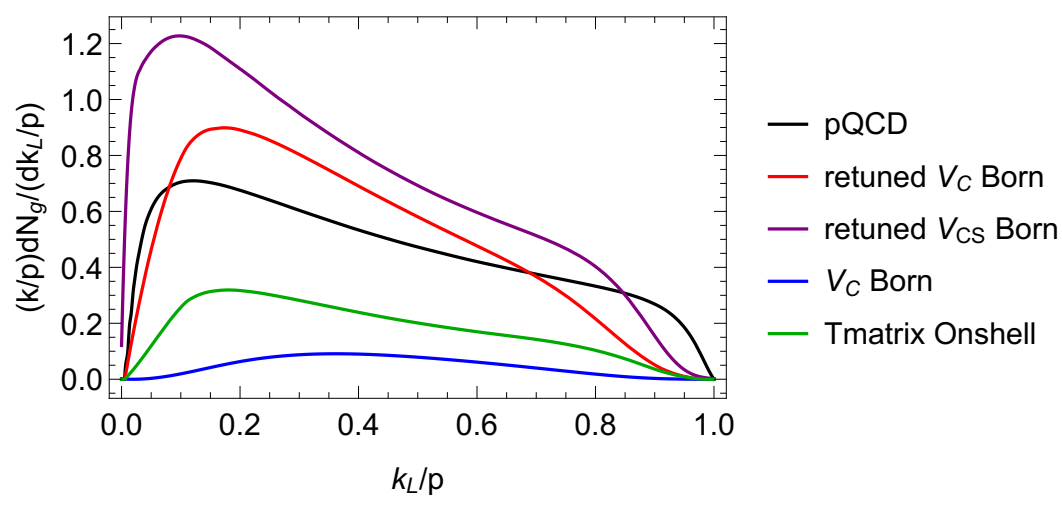

Figure 13. The quantity $\frac{(k / p) d N}{d\left(k_{L} / p\right)} \approx \frac{x d N}{d x}$ as a function of $k_{L} / p$. The initial HQ energy is $20 \mathrm{GeV}$ for a static medium of temperature $T=225 \mathrm{MeV}$ and length $L=5 \mathrm{fm}$. The pQCD result is taken from ref. [51].

state, as elaborated in section 3.1. In other words, the emitted gluon can only propagate in the energy momentum modes supported by the ambient medium. This is quite different from approaches where the emitted gluon in the QGP is assumed to be massless. The NP effects recover a good portion of the suppression in the power spectra, as we will discuss in more detail in the following section.

\subsection{Comparison to a pQCD calculation}

We finally carry out a more quantitative comparison of our results to a pQCD calculation from the literature [51]. Toward this end we focus on the quantity $x d N / d x$ commonly displayed in pQCD approaches, which characterizes the energy loss spectrum. We obtain this by integrating the power spectrum, $x d N / d x=\int d t x d N /(d t d x)$, over time as obtained from our Langevin simulation in section 3.3 which accounts for the time dependence of the charm-quark momentum, $p(t)$. In general, $x d N / d x$ is approximately equal to $x d N /(d t d x)$ multiplied by the length of the medium. We choose a QGP brick of length $L=5 \mathrm{fm}$ and temperature $T=0.225 \mathrm{GeV}$, and initial in charm-quark momentum of $20 \mathrm{GeV}$. For a most direct comparison of our calculations to the pQCD approach, we evaluate our " $V_{C}$ Born" scenario with the same parameters as in ref. [51], i.e., coupling constant $\alpha_{s}=0.3$, Coulomb Debye mass $m_{d}=g \sqrt{1+N_{f} / 6}$ with $N_{f}=2.5$, thermal light-quark and gluon masses of $m_{q}=m_{d} / \sqrt{6}$ and $m_{g}=m_{d} / \sqrt{2}$, respectively, and charmquark mass $m_{c}=1.2 \mathrm{GeV}$. At $T=0.225 \mathrm{GeV}$, this amounts to $m_{d}=0.51 \mathrm{GeV}, m_{q}=0.21 \mathrm{GeV}$, $m_{g}=0.36 \mathrm{GeV}$ and $m_{c}=1.2 \mathrm{GeV}$. The result of this retuned case agrees with the pQCD result taken from ref. [51] within $\sim 25 \%$, except for $x$ values close to 1 where our results go to zero faster, see figure 13. This comparison gives us further confidence that contributions from gauge-invariant pieces that are missing in our approach are not large, and that the comparisons of the different NP effects (which are much larger) are meaningful. In particular, if we include in the retuned set-up the string interaction (in Born approximation), we find a rather significant enhancement over the Coulomb-only scenario, as before. On the other hand, if we use our baseline parameters for the " $V_{C}$ Born" case $\left(\alpha_{s}=0.27, N_{f}=3\right.$, 
$m_{d}=0.42 \mathrm{GeV}, m_{q}=0.43 \mathrm{GeV}, m_{g}=1.15 \mathrm{GeV}$ and $\left.m_{c}=1.76 \mathrm{GeV}\right)$, the resulting energy loss spectrum is much reduced, cf. figure 13 . While the interaction strength is quite similar to the retuned scenario, the much larger quasiparticle masses fixed by fitting to the 1QCD EoS reduce the color charge density and with it the rate of radiation. In addition, the larger gluon and charm-quark masses (the latter as constrained by the HQ free energy) suppress the phase space for radiation. If we include the NP effects, i.e., the string interactions and resummations, the energy loss spectrum substantially increases over the " $V_{C}$ Born" result but is still significantly below the pQCD calculation. ${ }^{2}$ The large interaction strength cannot compensate the loss caused by the large NP masses, which are largely generated by the (remnants of the) confining force in our approach.

\section{Conclusion}

We have analyzed the radiative energy loss of heavy quarks within a thermodynamic $T$ matrix approach, on the same footing with earlier studies of collisional energy loss. The most relevant aspects of this calculation are its nonperturbative components, specifically remnants of the confining force above $T_{\mathrm{pc}}, t$-channel ladder resummations and off-shell spectral functions. These have been previously constrained by various sets of lattice-QCD data, most notably the QGP equation of state and heavy-quark free energy. To scrutinize their relevance, we have set up four scenarios with a subsequently increasing degree of NP effects.

We have found that all three NP effects referred to above substantially affect the power spectra and radiative transport coefficients at soft energy scales, i.e., at low $\mathrm{HQ}$ and/or gluon momenta and low temperatures. Ranking them by their importance, the inclusion of string interactions (even in Born approximation) generates the largest enhancement, followed by $t$-channel resummations in the two-body $T$-matrix, while the off-shell medium induces comparatively small modifications. Furthermore, we explicitly showed that the NP effects become gradually suppressed with increasing resolution scale; e.g., at $T=0.4 \mathrm{GeV}$ the NP enhancement in the radiative drag coefficient amounts to less than 40(20)\% for charm quarks of momenta of $\sim 20(40) \mathrm{GeV}$. This supports the convergence of pQCD-based approaches at high parton energies. While our calculations are carried out in a fixed gauge, we have checked that, when using color-Coulomb Born interactions and matching the input parameters to state-of-the-art pQCD calculations, our results for energy loss spectra for a high-energy charm quark agree with the latter within $\sim 25 \%$ or so. However, when using the thermal parton masses as dictated by the constraints from lattice-QCD, our baseline "pQCD" calculation results in a strongly suppressed radiation spectrum, mostly due to the large energy cost (mass) of the thermal modes available to the radiated gluons in the ambient QGP medium. The combined enhancement effect of the NP interactions cannot fully recover this suppression. It will thus be important to further srutinize the robustness of this result, and investigate its impact on in the phenomenology of HF observables in URHICs.

\footnotetext{
${ }^{2}$ As indicated in section 3.2, the drag coefficient from the "T-matrix Onshell" case is rather close to the "T-matrix Offshell" case, while the latter is computationally much more intensive.
} 


\section{Acknowledgments}

We are grateful for helpful discussions with Shanshan Cao and Yi-Lun Du. This work has been supported by the U.S. National Science Foundation (NSF) through grant no. PHY-1913286.

Open Access. This article is distributed under the terms of the Creative Commons Attribution License (CC-BY 4.0), which permits any use, distribution and reproduction in any medium, provided the original author(s) and source are credited.

\section{References}

[1] A. Dainese et al., Heavy ions at the Future Circular Collider, CERN Yellow Rep. (2017) 635 [arXiv: 1605.01389] [INSPIRE].

[2] F. Prino and R. Rapp, Open heavy flavor in QCD matter and in nuclear collisions, J. Phys. G 43 (2016) 093002 [arXiv: 1603.00529] [InSPIRE].

[3] B. Svetitsky, Diffusion of charmed quarks in the quark-gluon plasma, Phys. Rev. D 37 (1988) 2484 [INSPIRE].

[4] G.D. Moore and D. Teaney, How much do heavy quarks thermalize in a heavy ion collision?, Phys. Rev. C 71 (2005) 064904 [hep-ph/0412346] [INSPIRE].

[5] H. van Hees, V. Greco and R. Rapp, Heavy-quark probes of the quark-gluon plasma at RHIC, Phys. Rev. C $\mathbf{7 3}$ (2006) 034913 [nucl-th/0508055] [INSPIRE].

[6] X. Dong, Y.-J. Lee and R. Rapp, Open heavy-flavor production in heavy-ion collisions, Ann. Rev. Nucl. Part. Sci. 69 (2019) 417 [arXiv:1903.07709] [INSPIRE].

[7] M.G. Mustafa, Energy loss of charm quarks in the quark-gluon plasma: collisional versus radiative, Phys. Rev. C 72 (2005) 014905 [hep-ph/0412402] [INSPIRE].

[8] P.B. Gossiaux, J. Aichelin, T. Gousset and V. Guiho, Competition of heavy quark radiative and collisional energy loss in deconfined matter, J. Phys. G 37 (2010) 094019 [arXiv: 1001.4166] [INSPIRE].

[9] S. Cao, G.-Y. Qin and S.A. Bass, Heavy-quark dynamics and hadronization in ultrarelativistic heavy-ion collisions: collisional versus radiative energy loss, Phys. Rev. C $\mathbf{8 8}$ (2013) 044907 [arXiv: 1308.0617] [inSPIRE].

[10] P.B. Arnold, G.D. Moore and L.G. Yaffe, Photon and gluon emission in relativistic plasmas, JHEP 06 (2002) 030 [hep-ph/0204343] [INSPIRE].

[11] N. Armesto, C.A. Salgado and U.A. Wiedemann, Medium induced gluon radiation off massive quarks fills the dead cone, Phys. Rev. D 69 (2004) 114003 [hep-ph/0312106] [INSPIRE].

[12] R. Baier, Y.L. Dokshitzer, A.H. Mueller, S. Peigne and D. Schiff, Radiative energy loss of high-energy quarks and gluons in a finite volume quark-gluon plasma, Nucl. Phys. B 483 (1997) 291 [hep-ph/9607355] [INSPIRE].

[13] B.G. Zakharov, Fully quantum treatment of the Landau-Pomeranchuk-Migdal effect in QED and QCD, JETP Lett. 63 (1996) 952 [hep-ph/9607440] [INSPIRE].

[14] M. Gyulassy, P. Levai and I. Vitev, Reaction operator approach to non-Abelian energy loss, Nucl. Phys. B 594 (2001) 371 [nucl-th/0006010] [INSPIRE]. 
[15] M. Djordjevic, M. Gyulassy and S. Wicks, The charm and beauty of RHIC and LHC, Phys. Rev. Lett. 94 (2005) 112301 [hep-ph/0410372] [INSPIRE].

[16] X.-N. Wang and X.-F. Guo, Multiple parton scattering in nuclei: parton energy loss, Nucl. Phys. A 696 (2001) 788 [hep-ph/0102230] [INSPIRE].

[17] A. Majumder, Hard collinear gluon radiation and multiple scattering in a medium, Phys. Rev. D 85 (2012) 014023 [arXiv:0912.2987] [INSPIRE].

[18] G. Ovanesyan and I. Vitev, An effective theory for jet propagation in dense QCD matter: jet broadening and medium-induced bremsstrahlung, JHEP 06 (2011) 080 [arXiv:1103.1074] [INSPIRE].

[19] Z.-B. Kang, F. Ringer and I. Vitev, Inclusive production of small radius jets in heavy-ion collisions, Phys. Lett. B $\mathbf{7 6 9}$ (2017) 242 [arXiv:1701.05839] [INSPIRE].

[20] B.-W. Zhang, E. Wang and X.-N. Wang, Heavy quark energy loss in nuclear medium, Phys. Rev. Lett. 93 (2004) 072301 [nucl-th/0309040] [INSPIRE].

[21] M. Djordjevic, Theoretical formalism of radiative jet energy loss in a finite size dynamical QCD medium, Phys. Rev. C 80 (2009) 064909 [arXiv:0903.4591] [INSPIRE].

[22] R. Abir and A. Majumder, Drag-induced radiative energy loss from semihard heavy quarks, Phys. Rev. C 94 (2016) 054902 [arXiv:1506.08648] [INSPIRE].

[23] Z.-B. Kang, F. Ringer and I. Vitev, Effective field theory approach to open heavy flavor production in heavy-ion collisions, JHEP 03 (2017) 146 [arXiv:1610.02043] [INSPIRE].

[24] Y.-L. Du, Y. He, X.-N. Wang, H. Xing and H.-S. Zong, Revisiting heavy quark radiative energy loss in nuclei within the high-twist approach, Phys. Rev. D 98 (2018) 054015 [arXiv: 1807.06917] [INSPIRE].

[25] J. Uphoff, O. Fochler, Z. Xu and C. Greiner, Elastic and radiative heavy quark interactions in ultra-relativistic heavy-ion collisions, J. Phys. G 42 (2015) 115106 [arXiv:1408.2964] [INSPIRE].

[26] S.K. Das, J.-E. Alam and P. Mohanty, Dragging heavy quarks in quark gluon plasma at the Large Hadron Collider, Phys. Rev. C 82 (2010) 014908 [arXiv: 1003.5508] [InSPIRE].

[27] J. Xu, J. Liao and M. Gyulassy, Bridging soft-hard transport properties of quark-gluon plasmas with CUJET3.0, JHEP 02 (2016) 169 [arXiv:1508.00552] [INSPIRE].

[28] S. Cao, T. Luo, G.-Y. Qin and X.-N. Wang, Heavy and light flavor jet quenching at RHIC and LHC energies, Phys. Lett. B 777 (2018) 255 [arXiv:1703.00822] [InSPIRE].

[29] W. Ke, Y. Xu and S.A. Bass, Linearized Boltzmann-Langevin model for heavy quark transport in hot and dense QCD matter, Phys. Rev. C 98 (2018) 064901 [arXiv: 1806.08848] [INSPIRE].

[30] R. Katz, C.A.G. Prado, J. Noronha-Hostler, J. Noronha and A.A.P. Suaide, DAB-MOD sensitivity study of heavy flavor $R_{A A}$ and azimuthal anisotropies based on beam energy, initial conditions, hadronization, and suppression mechanisms, Phys. Rev. C 102 (2020) 024906 [arXiv: 1906.10768] [INSPIRE].

[31] JET collaboration, Extracting the jet transport coefficient from jet quenching in high-energy heavy-ion collisions, Phys. Rev. C 90 (2014) 014909 [arXiv: 1312.5003] [INSPIRE].

[32] A. Beraudo et al., Extraction of heavy-flavor transport coefficients in QCD matter, Nucl. Phys. A 979 (2018) 21 [arXiv:1803.03824] [INSPIRE]. 
[33] S. Cao et al., Toward the determination of heavy-quark transport coefficients in quark-gluon plasma, Phys. Rev. C 99 (2019) 054907 [arXiv: 1809.07894] [INSPIRE].

[34] H. van Hees and R. Rapp, Thermalization of heavy quarks in the quark-gluon plasma, Phys. Rev. C 71 (2005) 034907 [nucl-th/0412015] [INSPIRE].

[35] S.Y.F. Liu and R. Rapp, Spectral and transport properties of quark-gluon plasma in a nonperturbative approach, Eur. Phys. J. A 56 (2020) 44 [arXiv: 1612.09138] [INSPIRE].

[36] S.Y.F. Liu and R. Rapp, T-matrix approach to quark-gluon plasma, Phys. Rev. C 97 (2018) 034918 [arXiv: 1711.03282] [inSPIRE].

[37] S.Y.F. Liu, M. He and R. Rapp, Probing the in-medium QCD force by open heavy-flavor observables, Phys. Rev. C 99 (2019) 055201 [arXiv:1806.05669] [INSPIRE].

[38] J.F. Gunion and G. Bertsch, Hadronization by color bremsstrahlung, Phys. Rev. D 25 (1982) 746 [INSPIRE].

[39] C.D. Roberts and A.G. Williams, Dyson-Schwinger equations and their application to hadronic physics, Prog. Part. Nucl. Phys. 33 (1994) 477 [hep-ph/9403224] [INSPIRE].

[40] C.D. Roberts and S.M. Schmidt, Dyson-Schwinger equations: density, temperature and continuum strong QCD, Prog. Part. Nucl. Phys. 45 (2000) S1 [nucl-th/0005064] [INSPIRE].

[41] P. Maris and C.D. Roberts, Dyson-Schwinger equations: a tool for hadron physics, Int. J. Mod. Phys. E 12 (2003) 297 [nucl-th/0301049] [INSPIRE].

[42] J.I. Kapusta and C. Gale, Finite-temperature field theory: principles and applications, Cambridge University Press, Cambridge, U.K. (2011) [inSPIRE].

[43] M.L. Bellac, Thermal field theory, Cambridge University Press, Cambridge, U.K. (2011) [INSPIRE].

[44] L.D. Landau and I. Pomeranchuk, Limits of applicability of the theory of bremsstrahlung electrons and pair production at high-energies, Dokl. Akad. Nauk Ser. Fiz. 92 (1953) 535 [INSPIRE].

[45] A.B. Migdal, Bremsstrahlung and pair production in condensed media at high-energies, Phys. Rev. 103 (1956) 1811 [INSPIRE].

[46] M. Gyulassy and X.-N. Wang, Multiple collisions and induced gluon bremsstrahlung in QCD, Nucl. Phys. B 420 (1994) 583 [nucl-th/9306003] [INSPIRE].

[47] B. Singh, A. Abhishek, S.K. Das and H. Mishra, Heavy quark diffusion in a Polyakov loop plasma, Phys. Rev. D 100 (2019) 114019 [arXiv:1812.05263] [INSPIRE].

[48] Y.L. Dokshitzer and D.E. Kharzeev, Heavy quark colorimetry of QCD matter, Phys. Lett. B 519 (2001) 199 [hep-ph/0106202] [INSPIRE].

[49] S.S. Gubser, Momentum fluctuations of heavy quarks in the gauge-string duality, Nucl. Phys. $B \mathbf{7 9 0}$ (2008) 175 [hep-th/0612143] [INSPIRE].

[50] Y. Xu, J.E. Bernhard, S.A. Bass, M. Nahrgang and S. Cao, Data-driven analysis for the temperature and momentum dependence of the heavy-quark diffusion coefficient in relativistic heavy-ion collisions, Phys. Rev. C 97 (2018) 014907 [arXiv:1710.00807] [InSPIRE].

[51] M. Djordjevic and U. Heinz, Radiative heavy quark energy loss in a dynamical QCD medium, Phys. Rev. C $\mathbf{7 7}$ (2008) 024905 [arXiv: 0705.3439] [INSPIRE]. 